QUARTERLY OF APPLIED MATHEMATICS

VOLUME LXV, NUMBER 1

MARCH 2007, PAGES 1-23

S $0033-569 \mathrm{X}(07) 01039-5$

Article electronically published on January 9, 2007

\title{
ACCURATE CALCULATION OF THE MODIFIED MATHIEU FUNCTIONS OF INTEGER ORDER
}

\author{
BY \\ ARNIE L. VAN BUREN (Cary, North Carolina) \\ AND \\ JEFFREY E. BOISVERT (NAVSEA Newport, Newport, Rhode Island)
}

\begin{abstract}
Several different expressions involving infinite series are available for calculating the radial (i.e., modified) Mathieu functions of integer order. Mathieu functions depend on three parameters: order, radial coordinate, and a size parameter that is chosen here to be either real or imaginary. Expressions traditionally used to calculate the radial functions result in inaccurate function values over some parameter ranges due to unavoidable subtraction errors that occur in the series evaluation. For many of the expressions the errors for small orders increase without bound as the size parameter increases. In the present paper, the subtraction error obtained using traditional expressions is explored with regard to parameter values. Included is a discussion of the Bessel function product series, which has an integer offset for the order of the Bessel functions that is traditionally chosen to be zero (or one). It is shown here that the use of larger offset values that tend to increase with increasing radial function order usually eliminates the subtraction errors. This paper identifies the expressions and evaluation procedures that provide accurate radial Mathieu function values. A brief discussion of the calculation of the angular functions of the first kind that appear in many of these expressions is included. The paper also gives a description of a Fortran computer program that provides accurate values of radial Mathieu functions together with the associated angular functions over extremely wide parameter ranges. This effort was guided by recent advancements in the calculation of prolate spheroidal functions. The Mathieu functions are a special case of spheroidal functions, resulting in a similarity of behavior in their evaluation.
\end{abstract}

1. Introduction. The scalar Helmholtz wave equation for steady waves is separable in elliptic cylindrical coordinates $(u, v, z)$, where $0 \leqslant u \leqslant \infty, 0 \leqslant v \leqslant 2 \pi$, and $-\infty \leqslant$ $z \leqslant \infty$. In this paper we will use the radial coordinate $\xi=\cosh u$, where $1 \leqslant \xi \leqslant \infty$, instead of the traditional coordinate $u$. Separated solutions for the angular coordinate $v$ are the even angular Mathieu functions of the first kind $c e_{n}(v, c)$ and of the second kind $f e_{n}(v, c)$ and the odd angular Mathieu functions of the first kind $s e_{n}(v, c)$ and

Received October 4, 2005.

2000 Mathematics Subject Classification. Primary 33E10. 
of the second kind $g e_{n}(v, c)$. Here $n=0,1,2, \ldots$, is the integer order. Corresponding solutions for the radial coordinate are the even radial Mathieu functions of the first kind $M c_{n}^{(11)}(\xi, c)$ and of the second kind $M c_{n}^{\sqrt{21}}(\xi, c)$ and the odd radial Mathieu functions of the first kind $M s_{n}^{(1)}(\xi, c)$ and of the second kind $M s_{n}^{(2)}(\xi, c)$. Here $c$ is the size parameter. Real values of $c$ correspond to wave-field contributions that are non-evanescent, while imaginary values correspond to evanescent contributions.

When $c$ is imaginary, one usually does not calculate the radial functions of the second kind directly since they are in general complex. One instead calculates the radial functions of the third kind $\left(M_{n}^{(3)}=M_{n}^{(1)}+i M_{n}^{(2)}\right)$ or the fourth kind $\left(M_{n}^{(4)}=M_{n}^{(1)}-i M_{n}^{(2)}\right)$, which are real for odd orders and imaginary for even orders. Excellent treatments of Mathieu functions are found in the books by McLachlan [1] and Meixner and Schäfke [2. A summary of many of the relevant formulas for these functions is given in [3] and [4]. The separated solutions for the $z$-coordinate are exponentials that are easy to compute accurately.

The solution of physical problems usually involves angular functions of the first kind and radial functions of the first and second kinds for $c$ real and of the third (or fourth kind) for $c$ imaginary. Interior problems may also require radial functions of the first kind for $c$ imaginary. Existing computer programs for calculating the radial functions utilize traditional expressions that fail to provide accurate results for some parameter ranges, often at low values of $n$ when $|c|$ becomes large. In this paper we will present expressions and procedures for obtaining accurate values of the radial functions over virtually all parameter ranges.

In a previous paper [5] we showed how to obtain accurate values of the radial prolate spheroidal functions of the first kind over virtually all parameter ranges. In [6] we described new procedures for calculating accurate values of the radial prolate spheroidal functions of the second kind over parameter ranges much wider than previously possible. In the present paper we successfully apply the procedures developed for prolate spheroidal geometry to the accurate calculation of radial Mathieu functions. This success is largely due to the fact that Mathieu functions are a special case in the general theory of spheroidal functions [3].

We can obtain the algebraic form of Mathieu's equation from the differential equation satisfied by both radial and angular prolate spheroidal functions. The same algebraic equation is obtained for both the angular and the radial Mathieu functions when $\xi$ and $\eta=\cos v$ are used as the independent variables. We set the azimuthal number $m$ in the spheroidal equation equal to $1 / 2$ and factor out of the solution the term $\left(\xi^{2}-1\right)^{-1 / 4}$ in the radial case and the term $\left(1-\eta^{2}\right)^{-1 / 4}$ in the angular case. This results in Mathieu's equation.

We begin the paper with a discussion of the angular Mathieu functions of the first kind and their evaluation using traditional expansions in sines or cosines. Included is a description of a novel method for ensuring that the eigenvalues and the associated expansion coefficients obtained using the iterative procedure developed independently by Bouwkamp [7] for spheroidal functions and Blanch 8] for Mathieu functions are the correct ones. 
We then explore subtraction errors in the calculation of the radial functions using traditional single Bessel function expansions. These errors occur when the sum of the positive terms in the series is nearly equal in magnitude to the sum of the negative terms. This produces a loss of accuracy in the computed function value equal to the number of leading decimal digits that are the same in the two partial sums. We use the subtraction error results to identify which of the single Bessel function expansions provide accurate values and the parameter ranges where they do so. We next explore subtraction errors in the calculation of the radial functions using expansions in products of Bessel functions. These expansions have an integer offset for the order of the Bessel functions that is traditionally chosen to be zero (or one). We show how the use of non-traditional offset values often eliminates the subtraction errors.

We use the results to identify expressions and associated procedures that can be used to calculate accurate values for the radial Mathieu functions and describe a Fortran computer program that does so for extremely wide parameter ranges. The paper concludes with the summary.

2. Angular functions of the first kind. The angular Mathieu functions of the first kind $c e_{n}(v, c)$ and $s e_{n}(v, c)$ are expressed [1] in terms of the corresponding cosine and sine functions by

$$
\begin{aligned}
& c e_{n}(v, c)=\sum_{r=0,1}^{\infty}, A_{r}^{n}(c) \cos (r v), \\
& s e_{n}(v, c)=\sum_{r=2,1}^{\infty} B_{r}^{n}(c) \sin (r v),
\end{aligned}
$$

where the prime sign on the summation indicates that $r=0,2,4, \ldots$ if $n$ is even and $r=1,3,5, \ldots$ if $n$ is odd. Obviously, in (2) there is neither a sine angular function for $n=0$ nor a term for $r=0$. Corresponding expressions for the derivatives of the angular functions are obtained by differentiating (11) and (2). The expansion coefficients $A_{r}^{n}$ in (11) satisfy a three-term recursion formula that relates successive coefficients $A_{r}^{n}, A_{r+2}^{n}$, and $A_{r+4}^{n}$ for given values of $n$ and $c$. Use of this to calculate the expansion coefficients requires a value for the separation constant or eigenvalue $a_{n}(c)$, which is chosen to ensure non-trivial convergent solutions for $c e_{n}(v, c)$.

The variational procedure developed by Bouwkamp and Blanch can provide accurate values for both the eigenvalue and the ratios of successive coefficients $A_{r+2}^{n} / A_{r}^{n}$. This procedure starts with an estimate for the eigenvalue and then iteratively computes corrections to the eigenvalue until convergence to the desired accuracy is achieved. The procedure always converges to an eigenvalue corresponding to the desired value of $c$ but not always to the one for the desired order $n$ although it will correspond to an order of the same parity as $n$. The key to success is the starting value. For some eigenvalues the starting value must be extremely close to ensure convergence to that eigenvalue. If the starting value is slightly too large, the procedure converges to $a_{n+2}$. If it is slightly too small, the procedure converges to $a_{n-2}$. We previously developed a procedure that 
ensures correct eigenvalues for prolate spheroidal functions. With a slight modification this procedure also works for Mathieu functions.

We use traditional starting values for $n=0,1,2$, and 3 based on polynomial approximations for $c<\sqrt{40}$ and asymptotic approximations for $c \geqslant \sqrt{40}[3$. For larger orders we compute starting values using third-order extrapolation from the four previous eigenvalues. The variational procedure appears to always converge to the correct eigenvalue for $n=0$ using these starting values. It does not always do so for higher orders. In order to ensure the correct eigenvalues for $n>0$, we first establish lower and upper bounds $L_{n}$ and $U_{n}$ for an interval that contains the desired eigenvalue and no others of the same parity. The value used for $L_{n}$ is the previous eigenvalue since the eigenvalues form a monotonically increasing sequence with increasing order $n$. We obtain suitable values for $U_{n}$ from the starting values $\alpha_{n}$ and previously computed eigenvalues as follows:

$$
\begin{aligned}
& U_{1}=\alpha_{1}+\left(\alpha_{1}-a_{0}\right), \quad n=1, \\
& U_{2}=\alpha_{2}+0.5\left(\alpha_{2}-a_{0}\right), \quad n=2, \\
& U_{3}=\alpha_{3}+0.5\left(\alpha_{3}-a_{1}\right), \quad n=3, \\
& U_{n}=\alpha_{n}+0.5\left(a_{n-2}-a_{n-4}\right), \quad n>3 .
\end{aligned}
$$

This choice is based on the somewhat regular spacing of the eigenvalues. It is of course not unique.

If the converged eigenvalue lies within the established interval, it is the correct one. If it is smaller than $L_{n}$, then $L_{n}$ is changed to the starting value $\alpha_{n}$ and $U_{n}$ is left unchanged. If it is larger than $U_{n}$, then $U_{n}$ is instead changed to $\alpha_{n}$ and $L_{n}$ is left unchanged. A new starting value is chosen at the midpoint $\left(L_{n}+U_{n}\right) / 2$ of the new interval, and the process is repeated until the correct eigenvalue is obtained.

The expansion coefficient ratios are also obtained from the variational procedure. We normalize the coefficients by requiring that $c e_{n}(c, v)$ has the same normalization factor as the cosine, namely $\pi$, resulting in the following relation:

$$
\begin{gathered}
2\left(A_{0}^{n}\right)^{2}+\sum_{r=2}^{\infty}\left[A_{r}^{n}(c)\right]^{2}=1, \quad n \text { even }, \\
\sum_{r=1}^{\infty}\left[A_{r}^{n}(c)\right]^{2}=1, \quad n \text { odd } .
\end{gathered}
$$

This normalization is numerically robust with no subtraction errors. It has the practical advantage of eliminating the need to numerically evaluate the normalization factor which is often encountered in problems involving expansions in angular Mathieu functions. It also ensures that the largest coefficient (in magnitude) is of the order of unity. This is either the coefficient $A_{n}^{n}$ or one near it in the sequence. The coefficients tend to decrease in magnitude as the index $r$ decreases or increases from $n$. The rate of decrease is smaller, the larger the value of $c$.

The expansion coefficients $B_{r}^{n}$ in (2) satisfy a different three-term recursion formula than $A_{r}^{n}$, resulting in different eigenvalues $b_{n}(c)$ and coefficients. The procedures described above can be modified to compute $b_{n}(c)$ and the associated coefficient ratios. Traditional polynomial and asymptotic approximations 3 are used for the starting values $\beta_{1}, \beta_{2}, \beta_{3}$, and $\beta_{4}$. Third-order extrapolation from the four previous eigenvalues is 
used to obtain the starting values for higher orders. Upper and lower bounds are given as follows:

$$
\begin{aligned}
& U_{2}=\beta_{2}+\left(\beta_{2}-b_{1}\right), \quad n=2, \\
& U_{3}=\beta_{3}+0.5\left(\beta_{3}-b_{1}\right), \quad n=3, \\
& U_{4}=\beta_{4}+0.5\left(\beta_{4}-b_{2}\right), \quad n=4, \\
& U_{n}=\beta_{n}+0.5\left(b_{n-2}-b_{n-4}\right), \quad n>4 .
\end{aligned}
$$

The corresponding normalization relation is given by:

$$
\sum_{r=1,2}^{\infty},\left[B_{r}^{n}(h)\right]^{2}=1, \quad n \text { even or odd }
$$

The angular functions and associated eigenvalues and expansion coefficients for $c$ imaginary are related to those for $c$ real. For $n$ even, the eigenvalues are the same for $c$ and $i|c|$. For $n$ odd, the eigenvalue $a_{n}(i|c|)$ is equal to $b_{n}(c)$ and the eigenvalue $b_{n}(i|c|)$ is equal to $a_{n}(c)$. The angular functions are related as follows:

$$
\begin{aligned}
& c e_{n}(v, i|c|)=(-1)^{n / 2} c e_{n}(\pi / 2-v, c), \quad n \text { even, } \\
& c e_{n}(v, i|c|)=(-1)^{(n-1) / 2} s e_{n}(\pi / 2-v, c), \quad n \text { odd, } \\
& s e_{n}(v, i|c|)=(-1)^{(n-2) / 2} s e_{n}(\pi / 2-v, c), \quad n \text { even, } \\
& s e_{n}(v, i|c|)=(-1)^{(n-1) / 2} c e_{n}(\pi / 2-v, c), \quad n \text { odd. }
\end{aligned}
$$

We use (8) to obtain angular function values for $c$ imaginary from the corresponding values calculated for $\mathrm{c}$ real.

We use the series (11) and (2) to compute the angular functions in order starting with $c e_{0}$ and $s e_{1}$. The required trigonometric functions are computed only once for each argument $v$ from recursion formulas that relate $\sin [(r+1) v]$ and $\cos [(r+1) v]$ to $\sin (r v)$ and $\cos (r v)$ with coefficients $\sin (v)$ and $\cos (v)$. We first sum the series forward, starting with the term for $r=n$, until convergence is achieved. We require two conditions to be met for convergence. First the magnitude of the last term included relative to the sum must be less than 10 to the power $(-d-1)$, where $d$ is the number of decimal digits available in floating point arithmetic (usually 15 for $64 \mathrm{bits}$ ). Second, the corresponding trigonometric function for that term, either $\cos (r v)$ or $\sin (r v)$, must be larger than 0.1 to avoid false convergence when near one of their roots. Coefficients for each term are obtained by multiplying previous coefficients by coefficient ratios. We then sum the series backwards starting with the term for $r=n-2$ until either convergence is achieved or the first term in the series is reached.

The series for $c e_{n}$ and $s e_{n}$ are well behaved everywhere when $|c|$ is less than about 5 . For larger values of $|c|$, however, significant subtraction errors can occur for values of $n$ less than the break point $n_{b}=\operatorname{int}[2|c| / \pi]$. Here int[..] denotes integer truncation. The subtraction error increases as $v$ approaches 0 and $\pi$ for $c$ real and as $v$ approaches $\pi / 2$ and $3 \pi / 2$ for $c$ imaginary. For a given value of $n<n_{b}$, the subtraction error increases with increasing $|c|$. For a given value of $c$, the subtraction error is greatest for $c e_{0}$ and $s e_{1}$, decreasing to zero digits as the order increases to $n_{b}$. The behavior of the series for the first derivatives of the angular functions with respect to $v$ obtained by differentiation of (11) and (2) is similar to that of the series for the functions. 
The loss of accuracy in the angular functions due to these subtraction errors is rarely a problem when solving physical problems since it is accompanied by a decrease in magnitude relative to $\cos (n v)$ and $\sin (n v)$. This usually results in a decrease in the relative contribution of these function values to the solution of physical problems to the same degree as the loss in accuracy. However, the angular functions and their first derivatives at $v=0$ and $\pi / 2$ do appear in traditional expressions for the radial functions in series of Bessel functions. Since some of these special values involve significant subtraction errors during computation, their appearance often determines which expressions to use to compute a given function.

In Figure 1 we show the subtraction error obtained for $c e_{n}(0, c)$ as a function of $n$ for several values of $c$ real. The calculations were done in double precision (64-bit arithmetic) with $15+$ decimal digits available. We note that the subtraction error exceeds 15 digits for small values of $n$ when $c$ is larger than about 40. The behavior of the first derivative $s e_{n}^{\prime}(0, c)$ is nearly identical to that shown here. There is virtually no subtraction error in computing $c e_{n}(\pi / 2, c)$ and $s e_{n}^{\prime}(c, \pi / 2)$ for $n$ even or in computing $c e_{n}^{\prime}(c, \pi / 2)$ and $s e_{n}(c, \pi / 2)$ for $n$ odd.

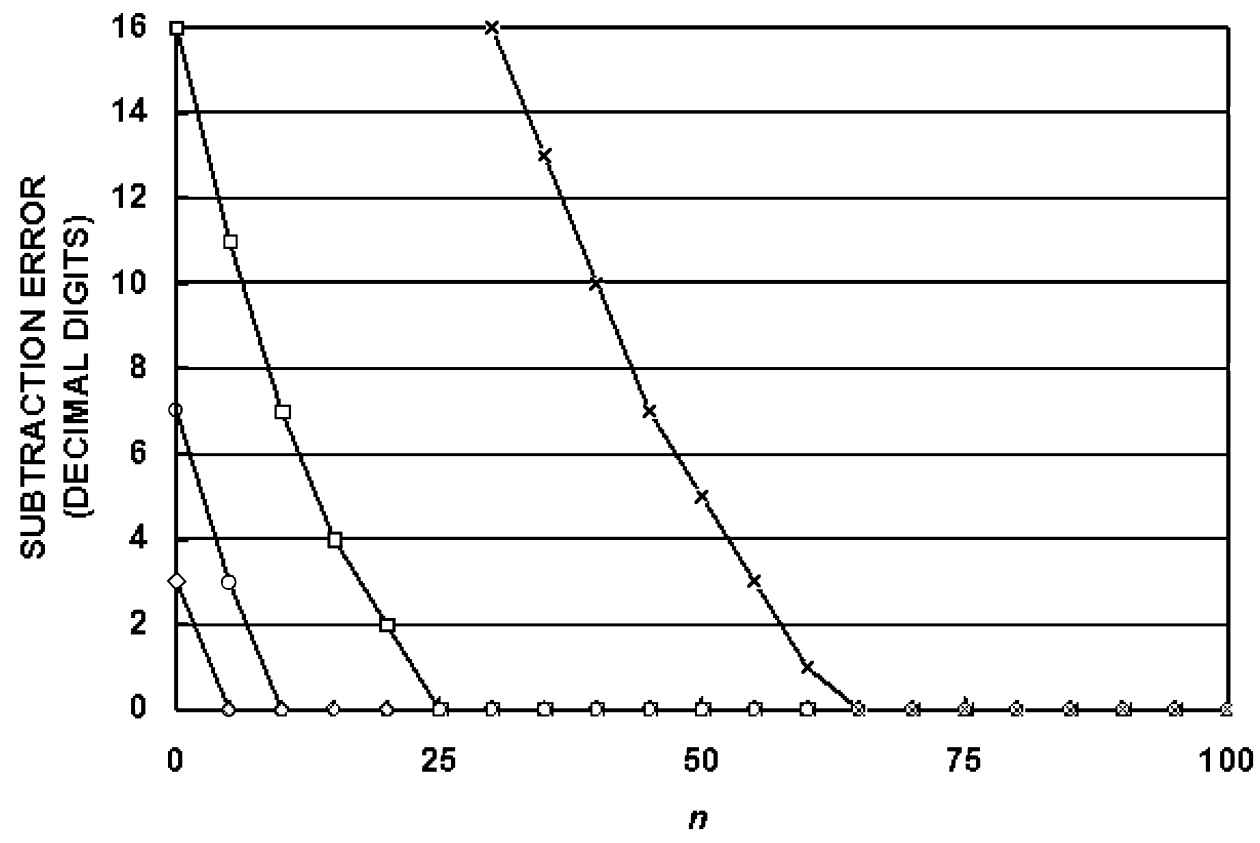

FIG. 1. Subtraction error in decimal digits in $c e_{n}(0, c)$ plotted versus $n$ for selected values of $c$ : $\diamond(10)$; o (20); $\square(40) ; \times(100)$.

3. Traditional single Bessel function expressions for the radial functions. The expansion of the product of the radial and the angular functions in terms of the 
corresponding cylindrical functions is given by:

$$
\begin{aligned}
& M c_{n}^{(j)}(\xi, c) c e_{n}(v, c)=\sum_{r=0,1}^{\infty} i^{r-n} A_{r}^{n}(c) \Phi_{r}^{(j)}(k r) \cos (r \theta), \\
& M s_{n}^{(j)}(\xi, c) s e_{n}(v, c)=\sum_{r=2,1}^{\infty} i^{r-n} B_{r}^{n}(c) \Phi_{r}^{(j)}(k r) \sin (r \theta) .
\end{aligned}
$$

Here $\Phi_{r}^{(j)}$ is the cylindrical Bessel function of the $j$ th type and $k$ is the wave number. This is a special case of the more general expansion given by Meixner and Schäfke [2, p. 177]. Using the relationship between the circular cylindrical coordinates $(r, \theta)$ and elliptic cylindrical coordinates (about the same origin and with $v=0$ coincident with $\theta=0)$, we have $k r=c\left(\xi^{2}+\cos ^{2} v-1\right)^{1 / 2}, \sin \theta=\sqrt{\xi^{2}-1} \sin v /\left(\xi^{2}+\cos ^{2} v-1\right)^{1 / 2}$, and $\cos \theta=\xi \cos v /\left(\xi^{2}+\cos ^{2} v-1\right)^{1 / 2}$. It is convenient to define $\eta=\cos v$. Substituting into (9) for $k r$ and $\theta$ and solving for $M c_{n}^{(j)}(\xi, c)$ and $M s_{n}^{(j)}(\xi, c)$, we have

$$
\begin{aligned}
M c_{n}^{(j)}(\xi, c)= & {\left[1 / c e_{n}(v, c)\right] } \\
& \times \sum_{r=0,1}^{\infty} i^{r-n} A_{r}^{n}(c) \Phi_{r}^{(j)}\left[c\left(\xi^{2}+\eta^{2}-1\right)^{1 / 2}\right] \cos \left[\xi \eta /\left(\xi^{2}+\eta^{2}-1\right)^{1 / 2}\right], \\
M s_{n}^{(j)}(\xi, c)= & {\left[1 / s e_{n}(v, c)\right] } \\
& \times \sum_{r=2,1}^{\infty} i^{r-n} B_{r}^{n}(c) \Phi_{r}^{(j)}\left[c\left(\xi^{2}+\eta^{2}-1\right)^{1 / 2}\right] \sin \left[\xi \eta /\left(\xi^{2}+\eta^{2}-1\right)^{1 / 2}\right] .
\end{aligned}
$$

The significance of these general expressions (10) is that we can choose the value for $v$ that provides the maximum accuracy for calculated values of $M c_{n}^{(j)}(\xi, c)$ and $M s_{n}^{(j)}(\xi, c)$. In [6] we showed how a similar expression for the prolate spheroidal radial functions leads to the traditional expressions for computing these functions. We also showed how it can be used to compute accurate values of the second kind $R_{m n}^{(2)}(\xi, c)$ over larger ranges than previously attainable by choosing progressively smaller values of $v$, starting with $\pi / 2$ and ending with 0 , in order to maintain accuracy as the order $n$ increases.

We can obtain the traditional Bessel function expansions found in the literature by setting $v=0$ or $v=\pi / 2$ in (10). Consider first the case for $v=0$. Here both the numerator and denominator vanish in the expression for $M s_{n}^{(j)}$, so we use L'Hospital's rule to compute the corresponding limits. The resulting expressions are:

$$
\begin{aligned}
& M c_{n}^{(j)}(\xi, c)=\left[1 / c e_{n}(0, c)\right] \sum_{r=0}^{\infty} \prime(-1)^{(r-n) / 2} A_{r}^{n}(c) \Phi_{r}^{(j)}(c \xi), \quad n \text { even }, \\
& M c_{n}^{(j)}(\xi, c)=\left[1 / c e_{n}(0, c)\right] \sum_{r=1}^{\infty} \prime(-1)^{(r-n) / 2} A_{r}^{n}(c) \Phi_{r}^{(j)}(c \xi), \quad n \text { odd }, \\
& M s_{n}^{(j)}(\xi, c)=\left[1 / s e_{n}^{\prime}(0, c)\right]\left[\sqrt{\xi^{2}-1} / \xi\right] \sum_{r=2}^{\infty}(-1)^{(r-n) / 2} r B_{r}^{n}(c) \Phi_{r}^{(j)}(c \xi), \quad n \text { even }, \\
& M s_{n}^{(j)}(\xi, c)=\left[1 / s e_{n}^{\prime}(0, c)\right]\left[\sqrt{\xi^{2}-1} / \xi\right] \sum_{r=1}^{\infty}(-1)^{(r-n) / 2} r B_{r}^{n}(c) \Phi_{r}^{(j)}(c \xi), \quad n \text { odd. }
\end{aligned}
$$

These expressions have limited use in computing the radial functions for $n<n_{b}$ when $c$ is real and large because of subtraction errors in both the series and in the angular functions appearing in the leading coefficients. The subtraction errors shown in Fig. 1 apply here. Significant subtraction errors also occur at higher orders in the series for the radial functions of the first kind for all values of real $c$, especially when $\xi$ is near 


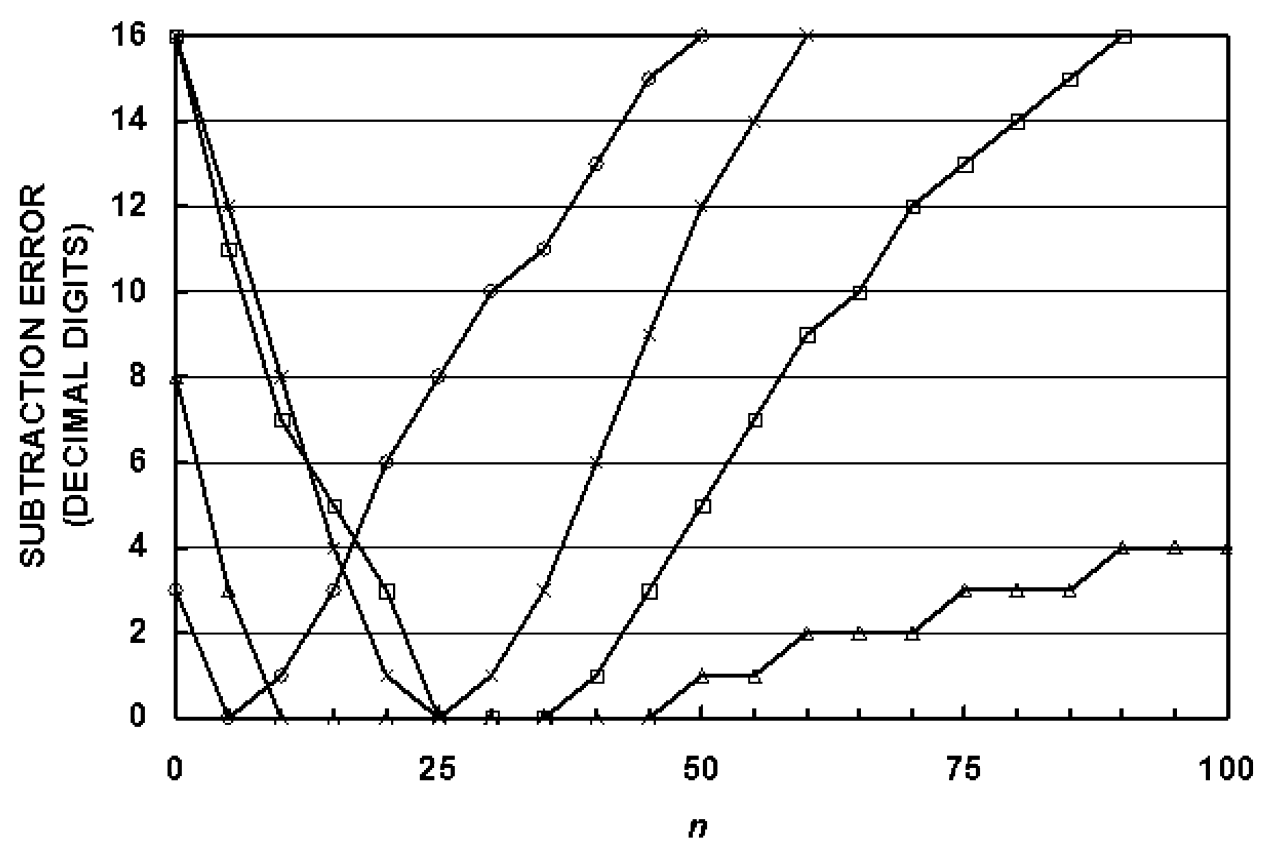

FIG. 2. Subtraction error in decimal digits in $M c_{n}^{(1)}(\xi, c)$ calculated using (11) plotted versus $n$ for selected values of $(\xi, c)$ : o $(1.001,10)$; $\times(1.001,40) ; \square(1.1,40) ; \Delta(2,20)$.

unity. Fig. 2 shows the subtraction errors in the series for $M c_{n}^{(\mathbb{1})}$ plotted as a function of $n$ for selected values of $\xi$ and $c$. The corresponding subtraction errors for $M s_{n}^{(1)}$ are similar.

When $c$ is imaginary, accurate values can now be obtained for the angular functions appearing in the leading coefficients in (11). For example, we can use the relations in (8) to replace the function $c e_{n}(0, i|c|)$ appearing in the expression for $M c_{n}^{(1)}$ with $n$ even by its equivalent $(-1)^{n / 2} c e_{n}(\pi / 2,|c|)$, which is accurately computed from (2). However, the radial functions of the first kind cannot be accurately calculated using these expressions when $c$ is imaginary and large unless $n$ is small because of significant subtraction errors occurring in the series. The Bessel functions appearing in (11) become the modified Bessel functions when $c$ is imaginary. The subtraction error for $M c_{n}^{(1)}$ is plotted in Fig. 3 as a function of $n$ for selected values of $\xi$ and $c$.

The radial functions of the third (and fourth kind), on the other hand, do not suffer subtraction errors (other than near a root) when computed using (11) with $c$ imaginary. Although the series tend to converge very slowly when $\xi$ is near unity, they do converge to accurate values. We found that the other traditional expressions do not provide accurate values for these functions for orders $n<n_{b}$ when $c$ is imaginary and large. We use the following expressions (12), obtained from (11) for $c$ imaginary, to compute $M c_{n}^{(3)}$ and 


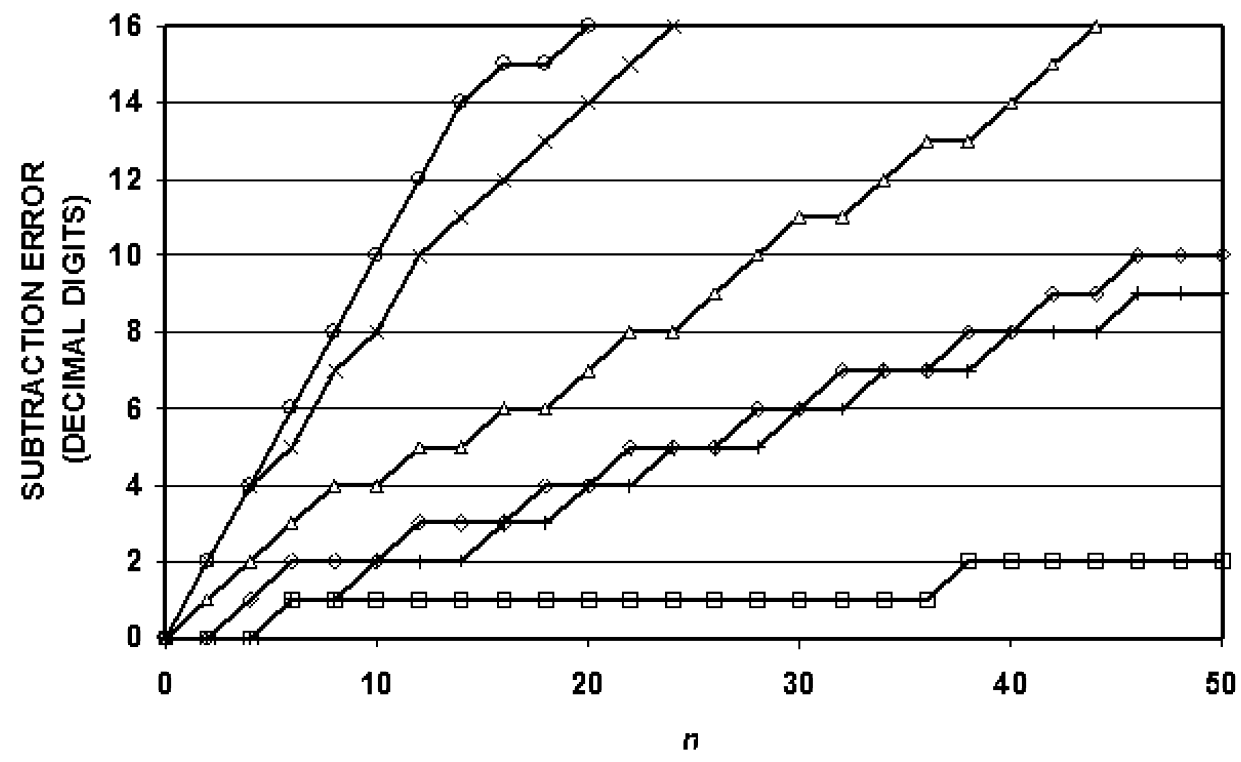

FIG. 3. Subtraction error in decimal digits in $M c_{n}^{10}(\xi, c)$ calculated using (11) plotted versus $n$ for selected values of $(\xi, c): \Delta(1.001,10 \mathrm{i}) ; \times(1.001,40 \mathrm{i})$; o $(1.001,100 \mathrm{i}) ; \diamond(1.1,10 \mathrm{i}) ; \square(2,10 \mathrm{i}) ;+(2,100 \mathrm{i})$.

$M s_{n}^{(3)}$ in this case:

$$
\begin{aligned}
M c_{n}^{(3)}(\xi, c)= & {\left[-2 i / \pi c e_{n}(\pi / 2,|c|)\right] \sum_{r=0}^{\infty}{ }^{(3)}(-1)^{(r-n) / 2} A_{r}^{n}(|c|) K_{r}(|c| \xi), n \text { even }, } \\
M c_{n}^{(3)}(\xi, c)= & {\left[-2 / \pi s e_{n}(\pi / 2,|c|)\right] \sum_{r=1}^{\infty}{ }^{\prime}(-1)^{(r-n) / 2} B_{r}^{n}(|c|) K_{r}(|c| \xi), n \text { odd }, } \\
M s_{n}^{(3)}(\xi, c)= & {\left[-2 i / \pi s e_{n}^{\prime}(\pi / 2,|c|)\right]\left[\sqrt{\xi^{2}-1} / \xi\right] } \\
& \times \sum_{r=2}^{\infty}(-1)^{(r-n) / 2} r B_{r}^{n}(|c|) K_{r}(|c| \xi), n \text { even, } \\
M s_{n}^{(3)}(\xi, c)= & {\left[2 / \pi c e_{n}^{\prime}(\pi / 2,|c|)\right]\left[\sqrt{\xi^{2}-1} / \xi\right] } \\
& \times \sum_{r=1}^{\infty}(-1)^{(r-n) / 2} r A_{r}^{n}(|c|) K_{r}(|c| \xi), n \text { odd } .
\end{aligned}
$$

The first derivatives $M c_{n}^{(3)^{\prime}}$ and $M s_{n}^{(3)^{\prime}}$ can be computed using expressions obtained from (12) by differentiation. We will describe in the next section how to obtain accurate values of $M c_{n}^{(3)}$ and $M s_{n}^{(\sqrt[3]{3})}$ for $n>n_{b}$ more efficiently than by using (12). Corresponding expressions for $M c_{n}^{(4)}$ and $M s_{n}^{(4)}$ are obtained from (12) by multiplying the expressions for even $n$ by -1 .

We compute the radial functions of the third kind from (12) using techniques described in [6] for $R_{m n}^{(2)}(\xi, c)$. First we factor out of the series the expansion coefficient and the Bessel function $K$ for $r=n$. We then sum the series forward from the $r=n$ term, 
starting with unity for $M c_{n}^{(3)}$ and $n$ for $M s_{n}^{(3)}$. Additional terms in the series are obtained by multiplying previous terms by both the ratios of successive Bessel functions and the ratios of successive expansion coefficients of the same parity as $n$. The first derivatives are computed at the same time using ratios of successive first derivatives of the Bessel functions of the same parity. These ratios of Bessel functions and their derivatives are computed once for the argument $c \xi$ using traditional recursion formulas. Convergence of the forward sum is achieved when the relative contribution of the last term to the sum has an absolute value less than 10 to the power $\left[-d-2-\log _{10}(|c|)\right]$ for both the radial function and its first derivative. This assures that the series will not be terminated prematurely when either a Bessel function or its first derivative happens to be near one of its roots. Here $d$ is again the number of decimal digits in double precision arithmetic. The term $\log _{10}(|c|)$ accounts for the tendency of the series to have an increasing number of successive terms in the series with the same sign as $|c|$ increases to large values.

The backward sum is calculated the same way as the forward sum except that division by ratios is used in place of multiplication. The resulting sum for both the radial function series and that for its first derivative are then multiplied by the value of the appropriate expansion coefficient and the characteristic for the Bessel function for $r=n$. The series for the first derivative is then multiplied additionally by the ratio of the first derivative to the function. These latter ratios were calculated once for each argument $c \sqrt{\xi^{2}-1}$ using a recursion formula developed for this purpose from standard recursion formulas. The Bessel function values for $r=n$ were calculated once for each argument using an exponent stripping procedure described in [5]. They consist of an integer exponent and a characteristic whose magnitude is near unity. We then multiply the sums by the appropriate angular function values and strip the exponent from the resulting product. This exponent is then added to the exponent for the Bessel function of order $n$. The radial functions of the third kind and their first derivatives are given by the resulting characteristics and integer exponents.

The series in (12) converge very slowly when $\xi$ is near unity and $c$ is large. The number of terms required for convergence increases with increasing order, with increasing $c$, and with decreasing $\xi-1$. Consider the case when $c$ is equal to 2000. Convergence to 10 decimal digits at $n=0$ requires 243 terms when $\xi=2 ; 13,201$ terms when $\xi=1.001$, and 110,920 terms when $\xi=1.0001$.

When we set $v=\pi / 2$ in (10), we obtain the second set of traditional expressions given for the radial functions. Here we use L'Hospital's rule for $M c_{n}^{(j)}$ when $n$ is odd and for $M s_{n}^{(j)}$ when $n$ is even. The resulting expressions are:

$$
\begin{aligned}
& M c_{n}^{(j)}(\xi, c)=\left[(-1)^{n / 2} / c e_{n}(\pi / 2, c)\right] \sum_{r=0}^{\infty} A_{r}^{n}(c) \Phi_{r}^{(j)}\left(c \sqrt{\xi^{2}-1}\right), n \text { even }, \\
& M c_{n}^{(j)}(\xi, c)=\left[(-1)^{(n+1) / 2} / c e_{n}^{\prime}(\pi / 2, c)\right]\left[\xi / \sqrt{\xi^{2}-1}\right] \sum_{r=1}^{\infty}{ }^{\prime} r A_{r}^{n}(c) \Phi_{r}^{(j)}\left(c \sqrt{\xi^{2}-1}\right), n \text { odd }, \\
& M s_{n}^{(j)}(\xi, c)=\left[(-1)^{n / 2} / s e_{n}^{\prime}(\pi / 2, c)\right]\left[\xi / \sqrt{\xi^{2}-1}\right] \sum_{r=2}^{\infty} r B_{r}^{n}(c) \Phi_{r}^{(j)}\left(c \sqrt{\xi^{2}-1}\right), n \text { even }, \\
& M s_{n}^{(j)}(\xi, c)=\left[(-1)^{(n-1) / 2} / s e_{n}(\pi / 2, c)\right] \sum_{r=1}^{\infty}{ }^{\prime} B_{r}^{n}(c) \Phi_{r}^{(j)}\left(c \sqrt{\xi^{2}-1}\right), n \text { odd. }
\end{aligned}
$$


All of the angular functions appearing in (13) are accurately calculated for $c$ real using (1) and (2). The series are also well behaved for $M c_{n}^{\mathbb{1}}$ and $M s_{n}^{\mathbb{1}}$ when $c$ is real. No subtraction errors occur unless $\xi$ is near a root. Corresponding expressions for $M c_{n}^{(\mathbb{1}) '}$ and $M s_{n}^{(1) \prime}$ obtained from (13) by differentiation are equally robust.

We must take care, however, when evaluating the series for $M c_{n}^{(\mathbb{1})^{\prime}}$ values of $\xi$ near unity when $n$ is odd and small. Consider the case for $n=1$ where the sum of the series tends to be dominated by the $r=1$ term. This term contains the factor $F=\xi^{2} w J_{1}^{\prime}(w)-J_{1}(w)$, where $w=c \sqrt{\xi^{2}-1}$. When $\xi$ is close to unity and $c$ is not too large, the two terms in this factor tend to cancel each other. Using power series representations for the Bessel functions, we obtain $F=(w / 2)-\left(3 w^{3} / 16\right)+\ldots-(w / 2)+\left(w^{3} / 16\right)-\ldots$, giving the approximation $F \approx-w^{3} / 8$. Calculation of this factor using the original expression produces a subtraction error in decimal digits approximately equal to $-\log _{10}\left[\left(w^{3} / 8\right) /(w / 2)\right]=$ $-\log _{10}\left(\xi^{2}-1\right)-2 \log _{10}(c / 2)$. As an example, the subtraction error is approximately 7 digits for $c=2$ and $\xi=1.0000001$. We can avoid this error by replacing the factor $F$ when $\xi$ is near unity with an equivalent expression that does not suffer subtraction errors. Using standard recursion relations, we obtain

$$
\begin{aligned}
F & =w J_{1}^{\prime}(w)-J_{1}(w)+\left(\xi^{2}-1\right) w J_{1}^{\prime}(w) \\
& =-w J_{2}(w)+\left(\xi^{2}-1\right) w J_{1}^{\prime}(w)
\end{aligned}
$$

which is numerically well behaved.

In [5] we showed that an expression similar to that given above in (13) for $M c_{n}^{(1)}$ provided accurate values for the prolate radial functions of the first kind $R_{m n}^{\mathbb{1}}(\xi, c)$. We also found similar subtraction errors in computing the first derivative for $m=0$ when $n$ is small and odd and $\xi$ is near unity. Now we realize that those errors can easily be eliminated using the method described above, which applies equally well to the calculation of $R_{0 n}^{\left(\mathbb{1 1}{ }^{\prime}\right.}(\xi, c)$.

We compute the series (13) for $M c_{n}^{(1)}$ and $M s_{n}^{\mathbb{1 1}}$ using techniques similar to those described in [5] for computing $R_{m n}^{\mathbb{1}}(\xi, c)$. These techniques differ in several ways from those described above for the computation of the series (12) for $M c_{n}^{(3)}$ and $M s_{n}^{(3)}$. We first sum the series in (13) backward from the $r=n$ term, starting with either unity or $n$. Additional terms in the series are obtained by dividing previous terms by ratios. The Bessel functions here have the argument $c \sqrt{\xi^{2}-1}$. Convergence of the backward sum is achieved if the first term in the series is reached or if the relative contribution of the last term to the sum has an absolute value less than 10 to the power $(-d-1)$ for both the radial function and its first derivative. For higher orders of the radial function when $\xi$ is near unity, the backward sum can become so large that the forward sum is not required. Scaling is used as necessary to avoid overflow in the sum of the backward series. If it is required, the summation is continued in the forward direction until convergence is obtained. The radial functions of the first kind and their first derivatives are given by the resulting characteristics and integer exponents. The exponents also include contributions from any scaling factors that may be used. 
When $c$ is imaginary we can use the following expressions (15), obtained from (13), to calculate $M c_{n}^{(1)}$ and $M s_{n}^{(10)}$ :

$$
\begin{aligned}
M c_{n}^{\mathbb{1}}(\xi, c)= & {\left[(-1)^{n / 2} / c e_{n}(0,|c|)\right] \sum_{r=0}^{\infty} A_{r}^{n}(|c|) I_{r}\left(|c| \sqrt{\xi^{2}-1}\right), \quad \text { n even }, } \\
M c_{n}^{(\mathbb{1})}(\xi, c)= & {\left[i(-1)^{(n-1) / 2} / s e_{n}^{\prime}(0,|c|)\right]\left[\xi / \sqrt{\xi^{2}-1}\right] } \\
& \times \sum_{r=1}^{\infty} r B_{r}^{n}(|c|) I_{r}\left(|c| \sqrt{\xi^{2}-1}\right), \quad n \text { odd }, \\
M s_{n}^{(\mathbb{1})}(\xi, c)= & {\left[(-1)^{n / 2} / s e_{n}^{\prime}(0,|c|)\right]\left[\xi / \sqrt{\xi^{2}-1}\right] } \\
& \times \sum_{r=2}^{\infty} r B_{r}^{n}(|c|) I_{r}\left(|c| \sqrt{\xi^{2}-1}\right), \quad n \text { even }, \\
M s \frac{10}{n}(\xi, c)= & {\left[i(-1)^{(n-1) / 2} / c e_{n}(0,|c|)\right] } \\
& \times \sum_{r=1}^{\infty} A_{r}^{n}(|c|) I_{r}\left(|c| \sqrt{\xi^{2}-1}\right), \quad n \text { odd. }
\end{aligned}
$$

However, the angular functions appearing in (15) suffer subtraction errors similar to those shown in Fig. 1. This restricts its use for calculating accurate values of $M c_{n}^{(1)}$ and $M s \frac{10}{n}$ to orders $n \geqslant n_{b}$ when $c$ is imaginary and large. Methods similar to those described above for real values of $c$ are used to evaluate (15) and the corresponding first derivative expressions obtained by differentiation.

The series in (13) are not very useful for calculating $M c_{n}^{(2)}$ and $M s_{n}^{(2)}$ since they converge absolutely and uniformly only if $\xi>\sqrt{2}[2$. We found that even when $\xi>\sqrt{2}$, accurate radial function values can usually only be obtained for small orders $n$ because of subtraction errors that increase rapidly with increasing order. Although the series for $M c_{n}^{(2)}$ and $M s_{n}^{(2)}$ diverge when $\xi<\sqrt{2}$, they appear to be asymptotic. As such they can provide accurate results when $c \sqrt{\xi^{2}-1}$ is very large if one uses the partial sum of the series up to and including the term where the relative contribution is a minimum. We were able to demonstrate this for $M c_{n}^{(2)}$ using techniques described in [6] for computing accurate values of the prolate radial spheroidal functions of the second kind.

4. Traditional Bessel product expressions for the radial functions. The Mathieu radial function can be expressed as an integral of the product of the corresponding angular function and a kernel function that is a solution to the Helmholtz equation [1]. Using kernels that are solutions in circular cylindrical coordinates $(r, \theta)$ produces:

$$
\begin{aligned}
& M c_{n}^{(j)}(\xi, c)=\lambda_{n}^{c} \int_{0}^{\pi} \Phi_{l}^{(j)}(k r) \cos (l \theta) c e_{n}(v, c) d v \\
& M s_{n}^{(j)}(\xi, c)=\lambda_{n}^{s} \int_{0}^{\pi} \Phi_{l}^{(j)}(k r) \sin (l \theta) s e_{n}(v, c) d v
\end{aligned}
$$

where $l$ is an arbitrary integer $\geqslant 0$ with the same parity as $n$. The coefficients $\lambda_{n}$ are characteristic values chosen so that the Mathieu radial functions have the same 
asymptotic limit as the corresponding cylindrical Bessel functions. The formulas relating $r$ and $\theta$ to the elliptic cylindrical coordinates $(\xi, v)$ are given in the previous section following (9).

In 6] we explored similar integral expressions for the radial spheroidal functions of the second kind $R_{m n}^{(2)}(\xi, c)$ in terms of the angular spheroidal functions $S_{m n}(\eta, c)$. The kernel function for $n-m$ even was the product of the spherical Neumann function $y_{m}(k R)$ and $\sin ^{m}(\vartheta)$, where $R$ is the spherical radius and $\vartheta$ is the spherical polar angle. This kernel is a solution to the Helmholtz equation in spherical coordinates, with the second factor being equal to a constant times the associated Legendre function $P_{m}^{m}(\vartheta)$. The kernel function for $n-m$ even was a constant times the product of $y_{m+1}(k R)$ and $P_{m+1}^{m}(\vartheta)$. We replaced $S_{m n}(c, \eta)$ in the integrand with its series expansion in $P_{r}^{m}(\eta)$. This resulted in a series of integrals of $P_{r}^{m}(\eta)$ times the kernel function. We used Gaussian quadrature to numerically evaluate the resulting integrals for given $\xi$ and $m$ over a wide range of values of $r$. A similar integral expression was used for $l-m$ odd. The radial spheroidal functions were then obtained for each desired order $n$ by summing the integrals over $r$ using the expansion coefficients appropriate for that order.

Numerical integration is not necessary when using (16) to compute Mathieu radial functions. Consider the case for $M c_{n}^{(j)}$. We can rewrite the Bessel function argument $k r=$ $c\left(\xi^{2}+\cos ^{2} v-1\right)^{1 / 2}$ as $\left(x_{1}^{2}+x_{2}^{2}-2 x_{1} x_{2} \cos \alpha\right)^{1 / 2}$, where $\alpha=\pi-2 v, x_{1}=c \exp (-z) / 2=$ $c\left(\xi-\sqrt{\xi^{2}-1}\right) / 2$, and $x_{2}=c \exp (z) / 2=c\left(\xi+\sqrt{\xi^{2}-1}\right) / 2$. This expression for $k r$ can be interpreted as the law of cosines for a triangle with sides $x_{1}, x_{2}$, and $k r$ and the angle $\alpha$ between the sides $x_{1}$ and $x_{2}$. Consider the angle $\chi$ between the sides $x_{1}$ and $k r$. Application of the law of sines gives $\sin \chi=\left(x_{2} \sin \alpha\right) / k r$. We can rewrite this as $\sin \chi=c\left(\xi-\sqrt{\xi^{2}-1}\right) \sin (\pi-2 v) / 2 k r=(c \xi \cos v / k r) \sin v-\left(c \sqrt{\xi^{2}-1} \sin v / k r\right) \cos v=$ $\cos \theta \sin v-\sin \theta \cos v=\sin (v-\theta)$. Similarly we can show that $\cos \chi=\cos (v-\theta)$ so that we have $\chi=v-\theta$.

The expression (16) for $M c_{n}^{(j)}$ now becomes

$$
\begin{aligned}
M c_{n}^{(j)}(\xi, c) & =\lambda_{n}^{c} \int_{0}^{\pi} \Phi_{l}^{(j)}(k r) \cos [l(v-\chi)] c e_{n}(v, c) d v \\
& \left.=\lambda_{n}^{c} \int_{0}^{\pi} \Phi_{l}^{(j)}(k r)[\cos l \chi \cos l v+\sin l \chi \sin l v)\right] c e_{n}(v, c) d v .
\end{aligned}
$$

We can apply Graf's addition theorem 3 for cylindrical Bessel functions to both $\Phi_{l}^{(j)}(k r) \cos l \chi$ and $\Phi_{l}^{(j)}(k r) \sin l \chi$ to obtain

$$
\begin{aligned}
M c_{n}^{(j)}(\xi, c) & \left.=\lambda_{n}^{c} \int_{0}^{\pi} \sum_{k=-\infty}^{k=\infty} J_{k}\left(x_{1}\right) \Phi_{k+l}^{(j)}\left(x_{2}\right)[\cos k \alpha \cos l v+\sin k \alpha \sin l v)\right] c e_{n}(v, c) d v \\
& =\lambda_{n}^{c} \sum_{k=-\infty}^{k=\infty} J_{k}\left(x_{1}\right) \Phi_{k+l}^{(j)}\left(x_{2}\right) \int_{0}^{\pi} \cos [k(\pi-2 v)-l v] c e_{n}(v, c) d v \\
& =\lambda_{n}^{c} \sum_{k=-\infty}^{k=\infty} J_{k}\left(x_{1}\right) \Phi_{k+l}^{(j)}\left(x_{2}\right) \int_{0}^{\pi}(-1)^{k} \cos [(2 k+l) v] c e_{n}(v, c) d v
\end{aligned}
$$


We replace $c e_{n}(v, c)$ with its expansion (1), interchange summations, and integrate term by term to obtain

$$
\begin{gathered}
M c_{n}^{(j)}(\xi, c)=\lambda_{n}^{c} \sum_{k=-\infty}^{k=\infty}(-1)^{k} J_{k}\left(x_{1}\right) \Phi_{k+l}^{(j)}\left(x_{2}\right) \int_{0}^{\pi} \sum_{r=0,1}^{\infty} A_{r} \cos [(2 k+l) v] \cos (r v) d v \\
=\lambda_{n}^{c} \sum_{r=0,1}^{\infty} A_{r} \sum_{k=-\infty}^{k=\infty}(-1)^{k} J_{k}\left(x_{1}\right) \Phi_{k+l}^{(j)}\left(x_{2}\right) \int_{0}^{\pi} \cos [(2 k+l) v] \cos (r v) d v \\
=\lambda_{n}^{c} \sum_{r=0,1}^{\infty} A_{r} \sum_{k=-\infty}^{k=\infty}(-1)^{k} J_{k}\left(x_{1}\right) \Phi_{k+l}^{(j)}\left(x_{2}\right)(\pi / 2)\left(\delta_{r, 2 k+l}+\delta_{r,-2 k-l)}\right) \\
=(\pi / 2) \lambda_{n}^{c} \sum_{r=0,1}^{\infty}{ }^{\prime} A_{r}\left[(-1)^{(r-l) / 2} J_{(r-l) / 2}\left(x_{1}\right) \Phi_{(r+l) / 2}^{(j)}\left(x_{2}\right)\right. \\
\left.\quad+(-1)^{-(r+l) / 2} J_{-(r+l) / 2}\left(x_{1}\right) \Phi_{-(r-l) / 2}^{(j)}\left(x_{2}\right)\right] \\
=(\pi / 2) \lambda_{n}^{c} \sum_{r=0,1}^{\infty}{ }^{\prime}(-1)^{(r-l) / 2} A_{r}\left[J_{(r-l) / 2}\left(x_{1}\right) \Phi_{(r+l) / 2}^{(j)}\left(x_{2}\right)\right. \\
\left.\quad+J_{(r+l) / 2}\left(x_{1}\right) \Phi_{(r-l) / 2}^{(j)}\left(x_{2}\right)\right] .
\end{gathered}
$$

When $n$, and consequently $l$, is even, we set $l=2 s$, where $s$ is an arbitrary integer $\geqslant 0$ to obtain:

$$
\begin{aligned}
M c_{n}^{(j)}(\xi, c)=(\pi / 2) \lambda_{n}^{c} \sum_{r=0}^{\infty}{ }^{\prime}(-1)^{(r / 2)-s} A_{r}[ & J_{(r / 2)-s}\left(x_{1}\right) \Phi_{(r / 2)+s}^{(j)}\left(x_{2}\right) \\
& \left.+J_{(r / 2)+s}\left(x_{1}\right) \Phi_{(r / 2)-s}^{(j)}\left(x_{2}\right)\right] .
\end{aligned}
$$

Choosing the coefficient $\lambda_{n}^{c}$ so that $M c_{n}^{(j)}$ has the same asymptotic behavior as $\Phi_{n}^{(j)}$ results in:

$$
\begin{aligned}
M c_{n}^{(j)}(\xi, c)=\left(1 / \varepsilon_{s} A_{2 s}\right) \sum_{r=0}^{\infty}{ }^{\prime}(-1)^{(r-n) / 2} A_{r}[ & J_{(r / 2)-s}\left(x_{1}\right) \Phi_{(r / 2)+s}^{(j)}\left(x_{2}\right) \\
& \left.+J_{(r / 2)+s}\left(x_{1}\right) \Phi_{(r / 2)-s}^{(j)}\left(x_{2}\right)\right]
\end{aligned}
$$

where $\varepsilon_{s}$ is equal to 2 if $s=0$ and equal to 1 otherwise.

We obtain the expression for $M c_{n}^{(j)}$ when $n$ is odd by setting $l=2 s+1$ in (19). We can derive the corresponding expressions for $M s_{n}^{(j)}$ by use of the same methods. We have:

$$
\begin{gathered}
M c_{n}^{(j)}(\xi, c)=\left[1 / A_{2 s+1}^{n}(c)\right] \sum_{r=1}^{\infty}{ }^{\prime}(-1)^{(r-n) / 2} A_{r}^{n}(c)\left[J_{[(r-1) / 2]-s}\left(x_{1}\right) \Phi_{[(r+1) / 2]+s}^{(j)}\left(x_{2}\right)\right. \\
\left.+J_{[(r+1) / 2]+s}\left(x_{1}\right) \Phi_{[(r-1) / 2]-s}^{(j)}\left(x_{2}\right)\right], n \text { odd }, \\
\begin{aligned}
M s_{n}^{(j)}(\xi, c)=\left[1 / B_{2 s}^{n}(c)\right] \sum_{r=2}^{\infty}{ }^{\prime}(-1)^{(r-n) / 2} B_{r}^{n}(c)\left[J_{(r / 2)-s}\left(x_{1}\right) \Phi_{(r / 2)+s}^{(j)}\left(x_{2}\right)\right. \\
+ \\
\left.\quad J_{(r / 2)+s}\left(x_{1}\right) \Phi_{(r / 2)-s}^{(j)}\left(x_{2}\right)\right], n \text { even }, \\
M s_{n}^{(j)}(\xi, c)=\left[1 / B_{2 s+1}^{n}(c)\right] \sum_{r=1}^{\infty},(-1)^{(r-n) / 2} B_{r}^{n}(c)\left[J_{[(r-1) / 2]-s}\left(x_{1}\right) \Phi_{[(r+1) / 2]+s}^{(j)}\left(x_{2}\right)\right. \\
\left.+J_{[(r+1) / 2]+s}\left(x_{1}\right) \Phi_{[(r-1) / 2]-s}^{(j)}\left(x_{2}\right)\right] . n \text { odd } .
\end{aligned}
\end{gathered}
$$




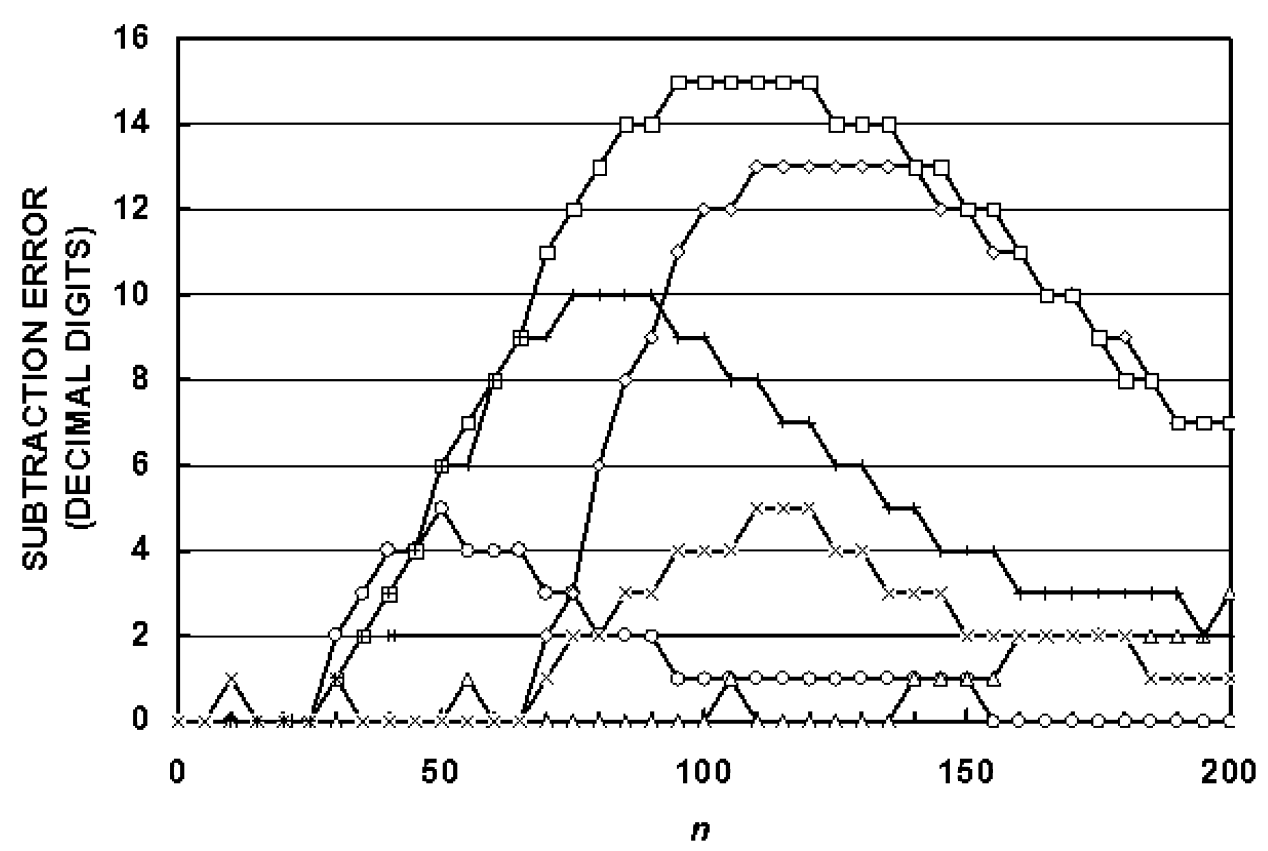

FIG. 4. Subtraction error in decimal digits in $M c_{n}^{(2)}(\xi, c)$ calculated using (21) and (22) with $s=0$ plotted versus $n$ for selected values of $(\xi, c): \Delta(1.001,200) ; \times(1.01,100) ;$ o $(1.1,40) ; \diamond(1.1,100)$; $\square(2,40) ;+(10,5)$.

These expressions are found in the literature [1, 2, 3, 9]. They appear to have been provided first by Dougall 9] who demonstrated their validity by direct substitution into Mathieu's equation for radial functions. Sieger [10] derived these expressions for the special case when $s$ is equal to 0 except for $M s_{n}^{(j)}$ with $n$ even when $s$ is equal to 1 . More recently, Meixner and Schäfke [2] derived the expressions for arbitrary $s$ using the generalized form of (9).

Researchers have traditionally used (21) and (22) to compute Mathieu radial functions with $s$ set equal to 0 except for $M s_{n}^{(j)}$ with $n$ even where $s$ is set equal to 1 . This choice can provide accurate radial function values for some parameter values, especially when $n$ and $c$ are not large. However, other parameter values can lead to significant subtraction errors. Figure 4 shows the resulting subtraction errors for $M c_{n}^{(2)}(\xi, c)$ plotted versus $n$ for selected values of $\xi$ and $c$ real. The errors are small when the order is somewhat smaller than the break point $n_{b}$. As the order increases beyond $n_{b}$, the subtraction error first increases until it reaches a maximum and then decreases to small values. For a given value of $\xi$, the maximum error increases with increasing $c$ while for a given value of $c$, the maximum error increases with increasing $\xi$. The corresponding subtraction errors for $M s_{n}^{(2)}$ are nearly identical to these. 


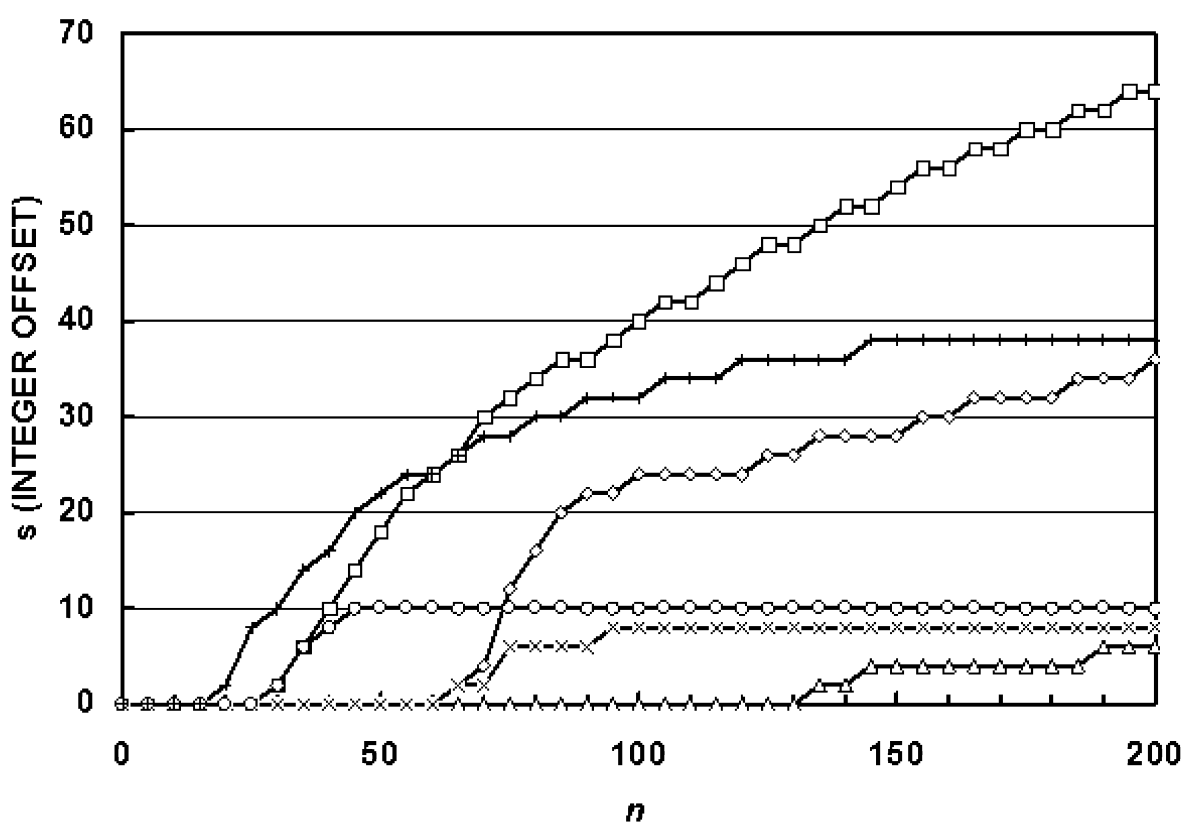

FIG. 5. Values of $s$ used in (21) and (22) for accurate $M c_{n}^{(2)}(\xi, c)$ plotted versus $n$ for selected values of $(\xi, c): \Delta(1.001,200)$; $\times(1.01,100) ;$ o $(1.1,40) ; \diamond(1.1,100) ; \square(2,40) ;+(10,5)$.

We can obtain significant improvement in calculating radial functions using (21) and (22) when we utilize $s$ as a free parameter chosen to minimize subtraction errors. For example, the errors shown in Fig. 4 for calculating $M c_{n}^{(2)}$ and $M s_{n}^{(2)}$ with $s$ equal to 0 or 1 can be reduced to zero or nearly so by using larger values for $s$ when the order $n$ is near or above the break point. We achieve this by using progressively larger values for $s$ as the order increases beyond the value $n^{\prime}=\operatorname{int}[0.6 c]$. The same values of $s$ work for both $M c_{n}^{(2)}$ and $M s_{n}^{(2)}$. The procedure is as follows. First $s$ is set equal to zero for $n=$ 0 or 1 . At each successive order we calculate the subtraction error nsub in the series for the radial functions and the corresponding subtraction error nsubd in the series for the first derivatives obtained from (18) by differentiation. When the order reaches the value $n^{\prime}$, the offset $s$ is incremented by $\Delta s=\max \left\{2, \operatorname{int}\left[\log _{10}(c)\right]\right\}$ if both nsub and nsubd for $M c_{n^{\prime}}^{(2)}$ exceed two digits. Here $\max \{. . .$.$\} denotes the larger of the two quantities. We$ then use the resulting value of $s$ for calculating $M s_{n^{\prime}}^{(\sqrt{2})}$. We increment $s$ again if both $n s u b$ and $n s u b d$ for $M s_{n^{\prime}}^{(2)}$ exceed two digits. The new value of $s$ is then used in the calculation of $M c_{n^{\prime}+1}^{(2)}$. The procedure is repeated until $n=n_{b}$ where $s$ is now incremented whenever both subtraction errors exceed zero digits. This new criterion is used for all higher orders. Figure 5 shows the values of $s$ that provide accurate values for $M c_{n}^{(2)}$ and $M s_{n}^{(2)}$ plotted versus $n$ for selected values of $\xi$ and $c$. 
We now consider the use of the Bessel product expansions for calculating $M c_{n}^{(1)}$ and $M s \frac{1}{n}$ when $c$ is imaginary. For this case (26) and (22) become:

$$
\begin{aligned}
& M c_{n}^{10}(\xi, c)=\left[(-1)^{s} / \varepsilon_{s} A_{2 s}^{n}(|c|)\right] \sum_{r=0}^{\infty} '(-1)^{(r-n) / 2} A_{r}^{n}(|c|)\left[I_{(r / 2)-s}\left(\left|x_{1}\right|\right) I_{(r / 2)+s}\left(\left|x_{2}\right|\right)\right. \\
& \left.+I_{(r / 2)+s}\left(\left|x_{1}\right|\right) I_{(r / 2)-s}\left(\left|x_{2}\right|\right)\right], n \text { even, } \\
& M c_{n}^{\mathbb{1}}(\xi, c)=\left[i(-1)^{s} / B_{2 s+1}^{n}(|c|)\right] \sum_{r=1}^{\infty} '(-1)^{(r-n) / 2} B_{r}^{n}(|c|) \\
& \times\left[I_{[(r-1) / 2]-s}\left(\left|x_{1}\right|\right) I_{[(r+1) / 2]+s}\left(\left|x_{2}\right|\right)\right. \\
& \left.+I_{[(r+1) / 2]+s}\left(\left|x_{1}\right|\right) I_{[(r-1) / 2]-s}\left(\left|x_{2}\right|\right)\right], n \text { odd, } \\
& M s_{n}^{11}(\xi, c)=\left[(-1)^{s} / B_{2 s}^{n}(|c|)\right] \sum_{r=2}^{\infty} '(-1)^{(r-n) / 2} B_{r}^{n}(|c|)\left[I_{(r / 2)-s}\left(\left|x_{1}\right|\right) I_{(r / 2)+s}\left(\left|x_{2}\right|\right)\right. \\
& \left.-I_{(r / 2)+s}\left(\left|x_{1}\right|\right) I_{(r / 2)-s}\left(\left|x_{2}\right|\right)\right], n \text { even, } \\
& M s \frac{11}{n}(\xi, c)=\left[i(-1)^{s} / A_{2 s+1}^{n}(|c|)\right] \sum_{r=1}^{\infty} '(-1)^{(r-n) / 2} A_{r}^{n}(|c|) \\
& \times\left[I_{[(r-1) / 2]-s}\left(\left|x_{1}\right|\right) I_{[(r+1) / 2]+s}\left(\left|x_{2}\right|\right)\right. \\
& \left.-I_{[(r+1) / 2]+s}\left(\left|x_{1}\right|\right) I_{[(r-1) / 2]-s}\left(\left|x_{2}\right|\right)\right], n \text { odd. }
\end{aligned}
$$

When $s$ is set equal to traditional values of 0 or 1 in (23), significant subtraction errors can occur. Figure 6 shows the resulting subtraction error for $M c_{n}^{(1)}$ plotted versus $n$ for selected values of $\xi$ and $c$. We can obtain values for $s$ that reduce the subtraction error to zero or nearly so by using a modified version of the approach described above for $c$ real. We discovered that the required values of $s$ are never less than the order and that they increase rapidly with increasing order. Thus we define $s=n+s^{\prime}$ and increment $s^{\prime}$ as the order increases, starting with $s^{\prime}=0$ for the lowest order. The value for $s^{\prime}$ is incremented whenever both subtraction errors nsub and nsubd exceed zero digits. The increment is given by

$$
\Delta s^{\prime}=1.5(n s u b+n s u b d)+\max \left\{0,2 \operatorname{int}\left[\log _{10}(|c|)\right]\right\}
$$

Figure 7 shows the value of $s$ that provides accurate values for $M c_{n}^{10}$ and $M s \stackrel{\text { (1) }}{\text { plotted }}$ versus $n$ for selected values of $\xi$ and $c$. When $|c|$ is large, the required value of $s$ for a given order $n$ tends to increase rapidly as $\xi$ approaches unity. For $\xi$ close to unity, the value of $s$ for a given order tends to increase rapidly as $|c|$ increases. The value of $s$ approaches the order $n$ as $|c|$ becomes small. We do not use (23) for orders greater than the break point $n_{b}$ since the expansions in Bessel functions of argument $|c| \sqrt{\xi^{2}-1}$ given in (15) converge rapidly and provide accurate values for this case. 


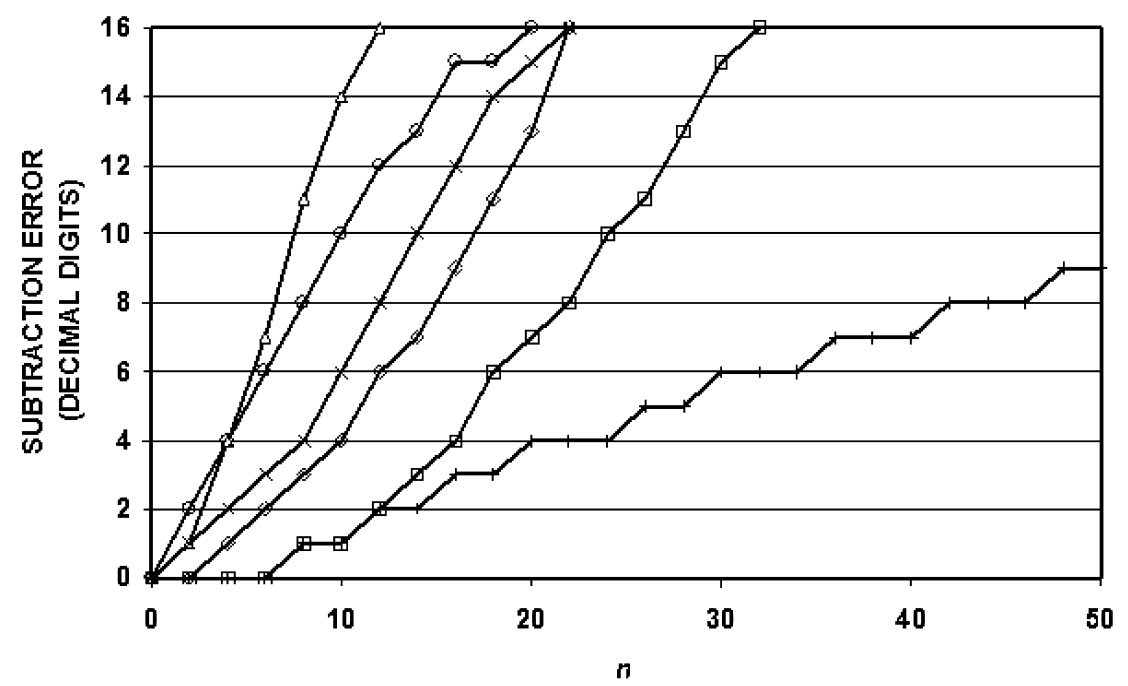

FIG. 6. Subtraction error in decimal digits in $M c_{n}^{\mathbb{1}}(\xi, c)$ calculated using (23) with $s=0$ plotted versus $n$ for selected values of $(\xi, c): \Delta(1.001, \mathrm{i})$; $\times(1.001,10 \mathrm{i})$; o $(1.001,100 \mathrm{i}) ; \diamond(1.1,10 \mathrm{i}) ; \square(2,10 \mathrm{i}) ;+(2,100 \mathrm{i})$.

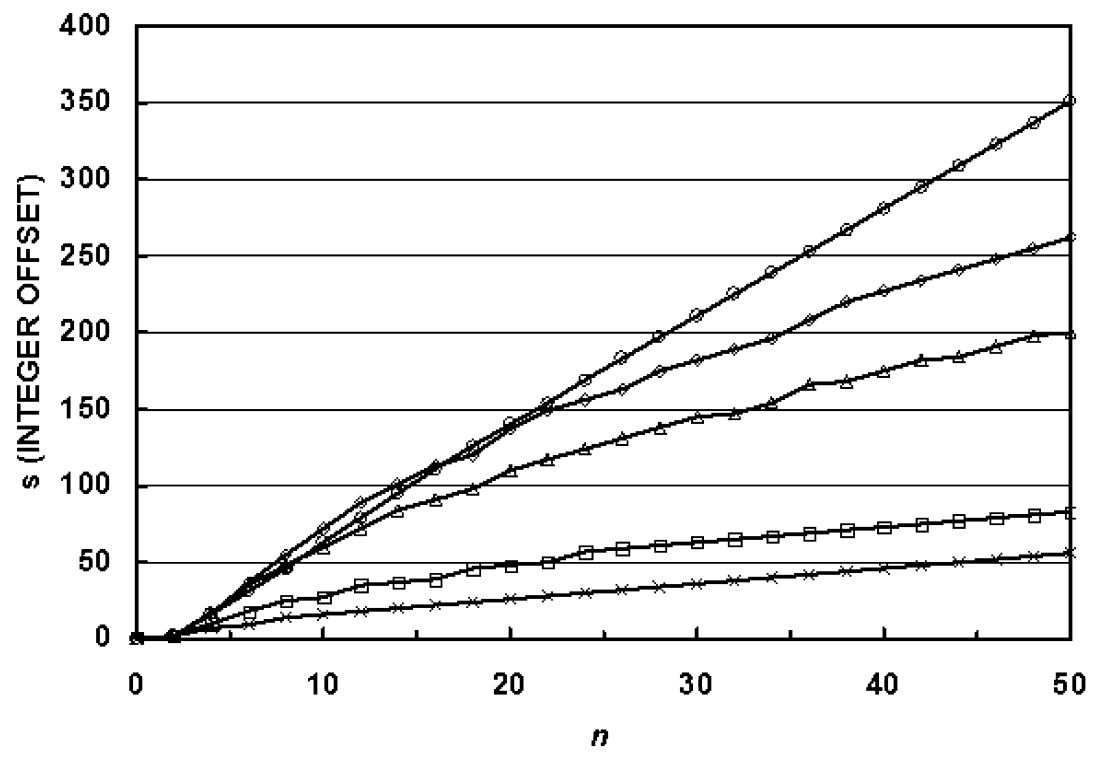

FIG. 7. Values of $s$ used in 23) for accurate $M c_{n}^{\mathbb{1}}(\xi, c)$ plotted versus $n$ for selected values of $(\xi, c): \times(1.001,10 \mathrm{i})$; o $(1.001,80 \mathrm{i})$; $\diamond(1.005,100 \mathrm{i}) ; \Delta(1.01,100 \mathrm{i}) ; \square(1.1,100 \mathrm{i})$. 


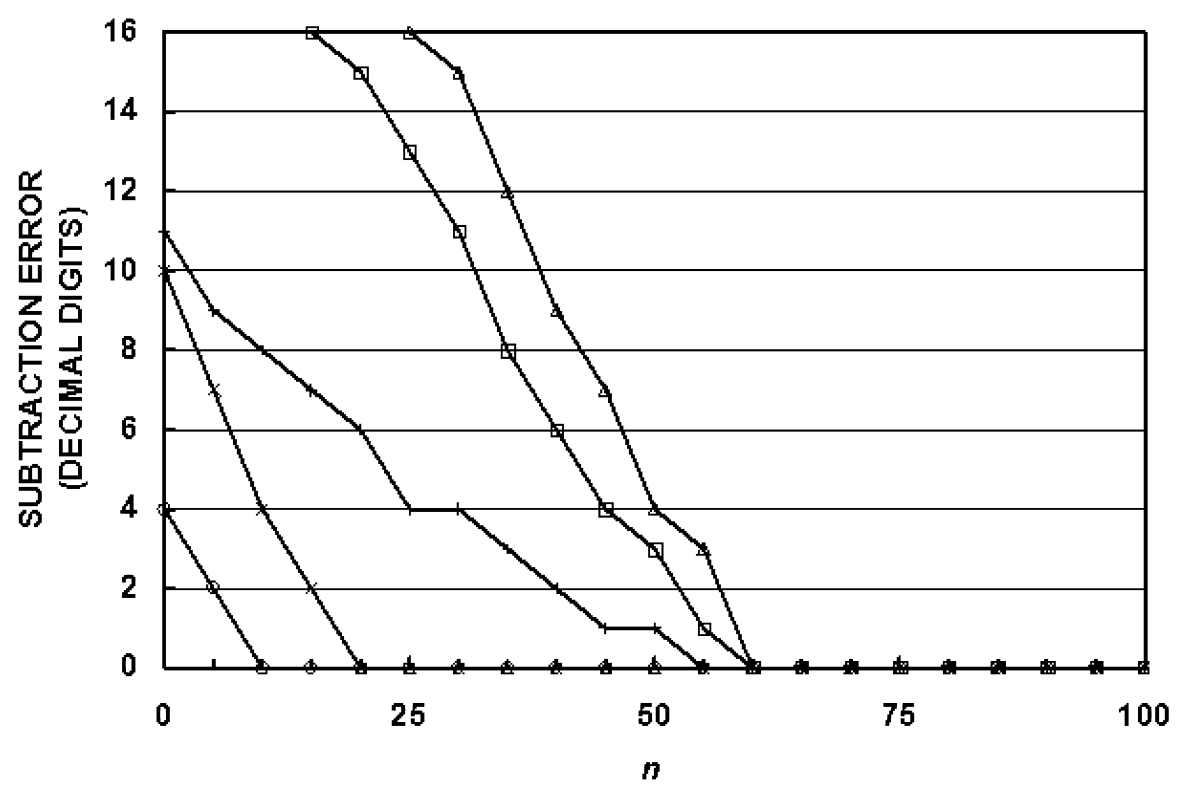

FIG. 8. Subtraction error in decimal digits in $M c_{n}^{[3]}(\xi, c)$ calculated using (24) with $s=0$ plotted versus $n$ for selected values of $(\xi, c)$ : $\Delta(1.001,100 \mathrm{i})$; o $(1.1,20 \mathrm{i}) ; \times(1.1,40 \mathrm{i}) ; \square(1.1,100 \mathrm{i}) ;+(2,100 \mathrm{i})$.

The following product expansions for $M c_{n}^{(3)}$ and $M s_{n}^{(3)}$ when $c$ is imaginary are obtained from (21) and (22):

$$
\begin{aligned}
& M c_{n}^{(3)}(\xi, c)=\left[-2 i(-1)^{n / 2} / \varepsilon_{s} \pi A_{2 s}^{n}(|c|)\right] \sum_{r=0}^{\infty}{ }^{\prime} A_{r}^{n}(|c|)\left[I_{(r / 2)-s}\left(\left|x_{1}\right|\right) K_{(r / 2)+s}\left(\left|x_{2}\right|\right)\right. \\
& \left.+I_{(r / 2)+s}\left(\left|x_{1}\right|\right) K_{(r / 2)-s}\left(\left|x_{2}\right|\right)\right], \quad n \text { even, } \\
& M c_{n}^{(3)}(\xi, c)=\left[2(-1)^{(n+1) / 2} / \pi B_{2 s+1}^{n}(|c|)\right] \sum_{r=1}^{\infty}{ }^{\prime} B_{r}^{n}(|c|)\left[I_{[(r-1) / 2]-s}\left(\left|x_{1}\right|\right) K_{[(r+1) / 2]+s}\left(\left|x_{2}\right|\right)\right. \\
& \left.-I_{[(r+1) / 2]+s}\left(\left|x_{1}\right|\right) K_{[(r-1) / 2]-s}\left(\left|x_{2}\right|\right)\right], \quad n \text { odd, } \\
& M s_{n}^{(3)}(\xi, c)=\left[-2 i(-1)^{n / 2} / \pi B_{2 s}^{n}(|c|)\right] \sum_{r=2}^{\infty}{ }^{\prime} B_{r}^{n}(|c|)\left[I_{(r / 2)-s}\left(\left|x_{1}\right|\right) K_{(r / 2)+s}\left(\left|x_{2}\right|\right)\right. \\
& \left.-I_{(r / 2)+s}\left(\left|x_{1}\right|\right) K_{(r / 2)-s}\left(\left|x_{2}\right|\right)\right], \quad n \text { even, } \\
& M s \stackrel{\sqrt{3})}{n}(\xi, c)=\left[2(-1)^{(n+1) / 2} / \pi A_{2 s+1}^{n}(|c|)\right] \sum_{r=1}^{\infty}{ }^{\prime} A_{r}^{n}(|c|)\left[I_{[(r-1) / 2]-s}\left(\left|x_{1}\right|\right) K_{[(r+1) / 2]+s}\left(\left|x_{2}\right|\right)\right. \\
& \left.+I_{[(r+1) / 2]+s}\left(\left|x_{1}\right|\right) K_{[(r-1) / 2]-s}\left(\left|x_{2}\right|\right)\right], \quad n \text { odd. }
\end{aligned}
$$

The subtraction errors in $M c_{n}^{(3)}$ and $M s_{n}^{(3)}$ calculated using (24) with traditional values of zero and one for $s$ are largest for the lowest order, decrease monotonically to zero as the order increases to the break point, and remain essentially zero for higher orders. We show in Fig. 8 the subtraction error obtained for $M c_{n}^{(3)}(\xi, c)$ plotted versus $n$ for selected values of $\xi$ and $c$. It turns out that this is the best we can do with (24). Larger values of $s$ increase the subtraction errors from those shown here. Use of the product expansion 
(24) for lower orders is thus limited to small values of $|c|$. We use instead the Bessel function expansions (12) to calculate accurate values for $M c_{n}^{(3)}$ and $M c_{n}^{(3)}$ when $n<n_{b}$. We use the product expansions (24) with traditional values of $s$ to obtain accurate values for $M c_{n}^{(3)}$ and $M c_{n}^{\left(\frac{3)}{3}\right)}$ when $n \geqslant n_{b}$. Corresponding expressions for the first derivatives obtained by differentiation of (24) work equally well.

We compute the series in (21)-(24) using methods similar to those described above for (12). First the required Bessel functions are calculated once for the two arguments $x_{1}$ and $x_{2}$ or $\left|x_{1}\right|$ and $\left|x_{2}\right|$ when $c$ is imaginary. Care must be taken to avoid subtraction errors when computing $x_{1}=c\left[\xi-\sqrt{\xi^{2}-1}\right] / 2$ for $\xi$ near unity. We use instead the equivalent expression $x_{1}=c /\left\{2\left[\xi+\sqrt{\xi^{2}-1}\right]\right\}$. We compare the magnitudes of the two Bessel function product terms appearing in the series for the term when $r=n$ and factor the larger one out of the series. The forward series is computed using ratios of Bessel functions and coefficients to obtain successive terms. Summation of the forward series continues until the relative contribution of the last term to the sum has an absolute value less than 10 to the power $(-d-1)$ for both the radial function and its first derivative. Then terms in the backward series are added until either the first term in the series is reached or convergence is achieved. The resulting radial functions are given as characteristics and integer exponents.

5. A computer program for calculating Mathieu functions. We have developed a Fortran computer program called matfcn to calculate the Mathieu angular and radial functions. It is written in double precision arithmetic and runs on a personal computer. Matfen has been tested extensively using a Fortran compiler that allocates $15+$ decimal digits and an exponent range of \pm 306 to double precision floating point numbers. It utilizes many of the computational methods developed previously [5, 6] for prolate spheroidal functions. This includes the use of ratios for Bessel functions and expansion coefficients and scaling techniques to avoid exponent overflow and underflow and to provide function values even when their exponents are extremely large in magnitude [5, 6].

Calculation options for matfcn include (1) $M c_{n}^{\sqrt[11]{1}}, M s_{n}^{\sqrt[(1)]{2}}, M c_{n}^{\sqrt[(2)]{n}}$, and $M s_{n}^{\sqrt[(2)]{n}}$ when $c$ is real or $M c_{n}^{(10)}, M s_{n}^{(1)}, M c_{n}^{(3)}$, and $M s_{n}^{(3)}$ when $c$ is imaginary, together with their first derivatives; (2) $c e_{n}$ and $s e_{n}$; and (3) $c e_{n}, c e_{n}$ together with their first derivatives. Both radial and angular functions can be calculated during the same run. This is more efficient than calculating them in separate runs since the expansion coefficients need only be computed once. In the literature first derivatives of the radial functions are usually defined with respect to the coordinate $u$ rather than with respect to $\xi=\cosh u$. For this reason we have written matfcn to provide derivatives with respect to $u$. It is easy to convert these values to derivatives with respect to $\xi$ by multiplying by $1 / \sqrt{\xi^{2}-1}$.

The output of the program includes diagnostic files (one for radial functions and one for angular functions) including information such as the number of terms both available and used in the various series and subtraction errors occurring in the series calculations. Matfcn provides an estimate of the number of accurate digits for the function values. It obtains the estimate for radial functions by comparing the theoretical values for their 
Wronskians with their values computed from numerical values for the radial functions and their first derivatives. The Wronskians are given for $c$ real by

$$
\begin{aligned}
& M c_{n}^{\left(\frac{11}{1}\right)} \frac{d}{d u} M c_{n}^{(2)}-M c_{n}^{(2)} \frac{d}{d u} M c_{n}^{(1)}=\frac{2}{\pi}, \\
& M s_{n}^{(1)} \frac{d}{d u} M s_{n}^{(2)}-M s_{n}^{(2)} \frac{d}{d u} M s_{n}^{(1)}=\frac{2}{\pi},
\end{aligned}
$$

and for $c$ imaginary by

$$
\begin{aligned}
& M c_{n}^{(1)} \frac{d}{d u} M c_{n}^{(3)}-M c_{n}^{\left(\frac{3}{3}\right)} \frac{d}{d u} M c_{n}^{(1)}=\frac{2 i}{\pi}, \\
& M s_{n}^{(1)} \frac{d}{d u} M s n_{n}^{(3)}-M s \frac{(3)}{n} \frac{d}{d u} M s_{n}^{(1)}=\frac{2 i}{\pi} .
\end{aligned}
$$

An estimate of the minimum number of accurate digits in each of the function and derivative values is given by the number of leading digits that agree in the computed and theoretical Wronskian values. This is a reasonable estimate except when one of the functions or its derivative is near a root so that its calculation involves subtraction errors. A second exception occurs when $c$ is real and large, $\xi$ is equal to or near unity, and the order $n$ is well below the break point. Here both $M c_{n}^{(2)}$ and the first derivative of $M s_{n}^{(2)}$ are much smaller in magnitude than $M s_{n}^{(2)}$ and the first derivative of $M c_{n}^{(2)}$, respectively. There are corresponding subtraction errors in their calculation that decrease as the order increases from 0 to the break point, increase as $\xi$ approaches unity, and increase without bound as $c$ increases. For both of these exceptions the affected values will be less accurate than the Wronskian estimate by about the number of digits of subtraction error. This loss of accuracy is rarely a problem when solving physical problems since it is usually accompanied by a decrease in the relative contribution of the less accurate values to the solution of physical problems to the same degree as the loss in accuracy.

The estimate of the accuracy of the angular functions and first derivatives is obtained by subtracting from 15 the sum of the number of digits of subtraction error in the series and two digits to account for possible inaccurate digits in either the expansion coefficients or the sine and cosine functions.

When $c$ is real, matfcn calculates the angular functions using (11) and (2). It calculates the radial functions of the first kind using the Bessel function expansions (13) and calculates the radial functions of the second kind using the Bessel product expansions (21) and (22) evaluated with variable $s$.

When $c$ is imaginary, matfcn calculates the angular functions using (11) and (2) and the formulas (8) that relate the angular functions with $c$ real to those with $c$ imaginary. It calculates the radial functions of the first kind for orders $n<n_{b}$ using the Bessel product expansions (23) with variable $s$ and for $n \geqslant n_{b}$ using the Bessel function expansions (15). Radial functions of the third kind are calculated for orders $n<n_{b}$ using the Bessel function expansions (12) and for $n \geqslant n_{b}$ using the Bessel product expansions (24) with traditional values for $s$.

We tested matfcn by calculating radial functions for the first 1001 values of $n$, unless $c$ was larger than 500 where we calculated the first $2 c+1$ values. Few if any applications will require Mathieu function values for orders greater than this, although matfcn should provide accurate values for higher order if required. We tested for $|c|$ in excess of 5000 and orders in excess of 10000. For real $c$ we used values of $\xi$ ranging from 1 to 1000. For imaginary $c$ we used values of $\xi$ ranging from 1.0001 to 1000 . We obtained at least 9 
digits of accuracy, except for functions or first derivatives very near one of their roots or for the special case of $M c_{n}^{(2)}$ and the first derivative of $M s_{n}^{(2)}$ when $c$ is real and large, $\xi$ is equal to or near unity, and the order $n$ is well below the break point. This should be sufficient accuracy for most applications.

When $c$ is real and less than about 1000, the series converge rapidly and the computation times are small. For example, calculation of the first 2001 orders of both functions and derivatives for $c$ equal to 1000 takes only about 1.5 seconds on a laptop computer with a $700 \mathrm{MHz}$ processor. Computation times ranged from 1.3 seconds for $\xi$ very close to unity (1.0000000001) to 1.6 seconds for $\xi$ equal to 100 . It takes only about 0.5 seconds when $c$ is equal to 100 and 1001 orders are calculated. The times go up somewhat when $c$ becomes very large. For example it takes about 30 seconds to compute 10001 orders for c equal to 5000 .

When $c$ is imaginary, the computation times are comparable to those for $c$ real if $\xi \geqslant 1.1$. However, the Bessel function expansion for the radial functions of the third kind can take hundreds of thousands of terms for convergence to 10 or more digits when $\xi$ is very close to unity and $c$ is large. This can significantly increase the computation times. For example, when $\xi$ is equal to 1.01 it takes 5 seconds for $c$ equal to 100 and 13 seconds for $c$ equal to 1000 .

6. Summary. We explored the ability of traditional expressions to calculate accurate values for Mathieu functions of integer order. Emphasis was on the subtraction errors that occur in some parameter ranges for all of the expressions. We used the results to determine how to calculate accurate values of radial Mathieu functions and their first derivatives. When the size parameter $c$ is real, the radial functions $M c_{n}^{(1)}$ and $M s_{n}^{(1)}$ are calculated using traditional Bessel function expansions (13) with argument $c \sqrt{\xi^{2}-1}$. We developed a new method for calculating $M c_{n}^{\sqrt[(2)]{2}}$ and $M s_{n}^{(2)}$ that is based on the general form of the Bessel product expansions (21) and (22). Here we use non-traditional values for the integer offset of the order of the Bessel functions to obtain accurate results.

When $c$ is imaginary, we calculate $M c_{n}^{(1)}$ and $M s_{n}^{10}$ for $n<n_{b}$ using Bessel product expansions (23) with non-traditional integer offsets and for $n \geqslant n_{b}$ using traditional Bessel function expansions (15) with argument $c \sqrt{\xi^{2}-1}$. The radial functions of the third kind $M c_{n}^{(3)}$ and $M s \frac{\sqrt{3})}{n}$ are calculated for $n<n_{b}$ using traditional Bessel function expansions (12) with argument $c \xi$ and for $n \geqslant n_{b}$ using Bessel product expansions (24) with traditional integer offsets.

We described a new Fortran computer program matfcn that uses these expressions and approaches to calculate accurate values for the radial Mathieu functions over extremely wide parameter ranges. Matfcn also provides values for the corresponding angular Mathieu functions. It utilizes many of the computational methods developed previously [5, 6] for prolate spheroidal functions. We successfully tested the program for values of $|c|$ in excess of 5000 and orders in excess of 10000. For real $c$ we used values of $\xi$ ranging from 1 to 1000 . For imaginary $c$ we used values of $\xi$ ranging from 1.0001 to 1000 . At least 9 digits of accuracy were obtained for the radial functions when computed in double precision (64-bit arithmetic), except for functions or first derivatives very near one of 
their roots or for the special case of $M c_{n}^{(2)}$ and the first derivative of $M s_{n}^{(2)}$ when $c$ is real and large, $\xi$ is equal to or near unity, and the order $n$ is well below the break point.

Acknowledgments. This work was supported by the Office of Naval Research Code 321 and the NAVSEA Newport ILIR Program.

\section{REFERENCES}

[1] N. W. McLachlan, Theory and Applications of Mathieu Functions, Dover Publications, New York, 1964. MR0174808 (30:5001)

[2] J. Meixner and F. W. Schäfke, Mathieusche Funktionen und Sphäroidfunktionen, Springer-Verlag, Berlin, 1954. MR0066500(16:586g)

[3] M. Abramowitz and I. A. Stegun, Handbook of Mathematical Functions with Formulas, Graphs, and Mathematical Tables, Dover, New York, 1966. MR0208797 (34:8606)

[4] S. Zhang and J. Jin, Computation of Special Functions, Wiley Interscience, New York, 1996. MR 1406797 (97m:65001)

[5] A. L. Van Buren and J. E. Boisvert, "Accurate calculation of prolate spheroidal radial functions of the first kind and their first derivatives," Quart. Appl. Math. 60, 589-599 (2002). MR1914443 (2003g:65025)

[6] A. L. Van Buren and J. E. Boisvert, "Improved calculation of prolate spheroidal radial functions of the second kind and their first derivatives," Quart. Appl. Math. 62, 493-507 (2004). MR2086042 (2005g:33047)

[7] C. J. Bouwkamp, "Theoretical and numerical treatment of diffraction through a circular aperture," IEEE Trans. Antennas and Propagation AP-18, 152-176 (1970). MR0277174(43:2911)

[8] G. Blanch, "On the computation of Mathieu functions," J. Math. Phys. 25, 1-20 (1946). MR0016690 $(8: 53 \mathrm{c})$

[9] J. Dougall, "The solution of Mathieu's differential equation," Proc. Edinburgh Math. Soc. 34, 176-196 (1916).

[10] B. Sieger, "Die Beugung einer ebenen elektrischen Welle an einem Schirm von eliptischen Querschnitt," Ann. Physik. 4, 626-664 (1908). 